\title{
Polymeric Hydrogels with Antimicrobial Activity-A Review of Their Progress
}

\author{
Na Peng ${ }^{1 *}$, Xiongzhi Zhang ${ }^{1}$, Huan $\mathrm{Xu}^{1}$ and $\mathrm{Yi} \mathrm{Liu}^{1,2 *}$ \\ ${ }^{1}$ Key Laboratory of Coal Conversion and New Carbon Materials of Hubei Province, School of Chemistry and Chemical Engineering, Wuhan \\ University of Science and Technology, Wuhan, Hubei 430081, China \\ ${ }^{2}$ College of Chemistry and Molecular Sciences, Wuhan University, Wuhan, Hubei 430072, China \\ *Corresponding author: Na Peng, Key Laboratory of Coal Conversion and New Carbon Materials of Hubei Province, School of \\ Chemistry and Chemical Engineering, Wuhan University of Science and Technology, Wuhan, Hubei 430081, China,
}

Yi Liu, College of Chemistry and Molecular Sciences, Wuhan University, Wuhan, Hubei 430072, China

\section{ARTICLE INFO}

Received: 幽 December 02, 2019

Published: 幽 December 16, 2019

Citation: Na Peng, Xiongzhi Zhang, Huan $\mathrm{Xu}$, Yi Liu. Polymeric Hydrogels with Antimicrobial Activity-A Review of Their Progress. Biomed J Sci \& Tech Res 23(5)-2019. BJSTR. MS.ID.003973.

Keywords: Hydrogel; Antimicrobial Activity; Wound Healing; Implant Scaffold

\section{ABSTRACT}

Microbial infections happened during wound healing and biomedical implant are serious health problem, which have driven the development of advanced materials with antimicrobial activity. Hydrogels possessing porous structure, swelling property, mechanical performance, and biocompatible nature make them idea platforms to fabricate antimicrobial materials. Here, recently developed hydrogels with antimicrobial activity are summarized and discussed, which can be categorized based on their antimicrobial source, i.e., hydrogels containing antimicrobial agents and inherent antimicrobial hydrogels. In each section, representative studies will be highlighted with emphasis on the fabrication and properties of hydrogels. Finally, challenges in the antimicrobial hydrogels and future directions in this research field will be briefly presented and prospected.

Abbreviations: NIR: Near-Infrared, GC: Glycol Chitosan, CIP: Hydrogel-Based Ciprofloxacin, PAA: Poly (Acrylic Acid), CNPS: Carbon Black Nanoparticles, GS: Gentamycin Sulfate, PEG: Poly Ethylene Glycol, AMPS: Antimicrobial Peptides,

\section{Introduction}

Microorganism such as bacteria, viruses, parasites, or fungi, plays a vital role in wound healing by affecting risk of infection, healing time, recovery time of patient, and costs to the health service [1]. Especially, infectious diseases caused by pathogenic microorganism have become major public health threats, which can prolong or impair the wound healing process leading to tissue morbidity, sepsis, and implant failure. Due to the emergence of clinical importance of anti-infectious biomaterials, amounts of researches have been focused on improving implant surfaces via coatings which could effectively prevent bacterial adhesion, kill bacteria on contact, or enable controlled elution of antimicrobial compounds [2]. On the other hand, the over prescription of antibiotics induced bacteria resistance to the antimicrobial agents which was also a very serious problem of human health [3]. Therefore, the development of novel antimicrobial agents as potential substitutes for conventional antibiotics and advanced

materials with antimicrobial activity have become urgent and has received significant research efforts.

Recently, hydrogels were considered as effective antimicrobial formulations which can be applied broadly in a variety of biomedical-associated contexts. Because antimicrobial hydrogels, which contained hydrated polymeric networks possessing the ability to eliminate infectious microbes, have been exploited in many pharmaceutical applications, including medication, disinfectants, sanitizers, and personal care products [4]. Clinical studies showed that hydrogels provided a moist environment around the wound site to improve the healing process by protecting damaged skin from cellular dehydration and angiogenesis [5]. The porous architecture of hydrogels provided channels for absorbing wound exudate and allowing air permeability $[6,7]$. Furthermore, hydrogels exhibited antimicrobial activity after incorporating antimicrobial agents or introducing cationic polymers into their 
networks [4,7]. These advantages made hydrogels suitable for skin infection treatment, medical implants coating, and wound healing.

So far, antimicrobial hydrogels can be divided into two types based on the origin of antimicrobial activity, i.e., hydrogel as a matrix for antimicrobial agents (e.g., Ag nanoparticles, antibiotics, organic compounds, etc.), and hydrogels with inherent antimicrobial activity (e.g., natural polymer based hydrogels, synthetic polymeric hydrogels, peptide based hydrogels, etc.). Hydrogel as a matrix for antimicrobial agents can also act as controlled release system to noncovalently encapsulate and deliver bioactive molecules. Hydrogels with inherent antimicrobial activity were typically polycationic and worked through non-stereospecific mechanisms that involve membrane disruption. There are a number of outstanding reviews have been published with regards to this topic $[1,3,4,7]$. In this review, we will give an overview of the recent progress in antimicrobial hydrogels. Then, we will highlight some of the recent representative research works with emphasis on their structure, and antimicrobial activity, focusing on their innovative points, and discuss their advantage and drawbacks. Finally, the challenges and future research direction in the field of antimicrobial hydrogels will be proposed.

\section{Hydrogel as A Matrix for Antimicrobial Agents}

One of the main applications for polymeric hydrogel is drug delivery, where hydrogel networks can be used as controlled release system for encapsulation of small molecules [8]. Therefore, antimicrobial agents including antibiotics, silver nanoparticles, other metal nanoparticles and compounds, as well as other organic compounds can also be incorporated into hydrogel networks which acted as matrices for construction of composite hydrogels with antimicrobial activity.

\section{Hydrogels Containing Antibiotics}

Antibiotics are considered as the most efficient medicines for fighting bacterial infections, where they can selectively kill bacteria although their overuse may lead to an increasing number of resistant pathogens. Penicillin, cephalosporin, and fluoroquinolone are top three widely used antibiotics, and others such as gentamicin, amikacin, colistin, amphotericin B, and ciprofloxacin, are also common clinical medicines [9]. Hydrogel networks can be prepared by chemical cross-linking [10,11]. physical cross-linking, [12] and irradiation cross-linking for controlled release of antibiotics to possess antibacterial properties [13]. Supercritical assisted atomization and spray-drying were also employed to prepare the hydrogel carriers for drug delivery [14]. Both synthetic and natural polymers could participate in the design of hydrogel networks for antibiotics-controlled release system.

With the aim to design and develop suitable materials for efficient and robust controlled drug delivery system, stimuli-sensitive hydrogels were fabricated as the matrices for antibiotics. Hydrogel carriers with temperature- and pH-responsive properties for anticancer and anti-inflammatory drugs were also prepared by UV cross-linking of carboxymethyl chitosan-g-poly ( $\mathrm{N}$-isopropyl acrylamide) (PNIPAAm)-glycidyl methacrylate. The release percentage of diclofenac sodium from the hydrogel was $27 \%$ in $\mathrm{pH} 2.1$ at $37^{\circ} \mathrm{C}$ for $24 \mathrm{~h}$, whereas it was $89 \%$ in $\mathrm{pH} 7.4$ solution at $37^{\circ} \mathrm{C}$ for $24 \mathrm{~h}$, which means the hydrogels could protect the drug in low $\mathrm{pH}$ medium (such as stomach) and substantially release them at higher $\mathrm{pH}$ condition (such as intestine) [15]. B. Wang et al. constructed xanthan gum-succinic anhydride hydrogels for the ionic strength-sensitive release of gentamicin, where the release of antibiotics sustained as long as 9 days under aqueous physiological conditions [16]. Poly (acrylic acid) (PAA) and p(2-(dimethylamino) ethyl methacrylate) (PDMAEMA) formed the pH-sensitive composite hydrogel, which could degrade in the weak acidic microenvironment of bacterial infection, and smartly release antibiotics to fight bacterial infection [17]. Similarly, pH-sensitive dual drug loaded hydrogels consisted of gelatin methacrylate and methacrylic acid were developed for the controlled release of gentamicin and ampicillin.

The in vitro release profiles suggest the $\mathrm{pH}$ dependent behavior of the hydrogel, and antimicrobial studies revealed their increased efficiency in killing bacteria [18]. Lately, a Near-Infrared (NIR) light-triggerable thermo-sensitive Hydrogel-Based Ciprofloxacin (Cip) reservoir containing Cip-loaded Polydopamine (PDA) nanoparticles via $\pi-\pi$ stacking interaction and Glycol Chitosan (GC) was fabricated. The Gel-Cip showed minimal leakage under physiological conditions but could boost Cip release in an on-demand way under NIR light irradiation. In addition, it was found that the excellent photothermal effect of PDA nanoparticles induced elevated temperature via NIR light irradiation could also destroy the surrounding bacterial cell surfaces, which exhibited a synergistic effect in killing of both Gram-positive and Gram-negative bacteria. More importantly, the in vivo experiments further confirmed that the combination treatment of Gel-Cip with NIR light irradiation had greatly reduced bacterial load in the infected sites, and effectively promoted the wound healing of bacteria-infected mice (Figure 1) [19].

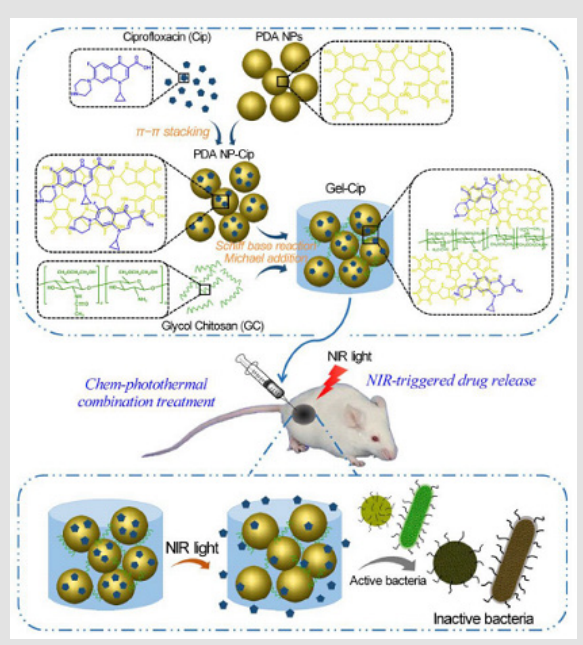

Figure 1: Schematics of the synthetic route of Gel-Cip and NIR light irradiation-triggered Cip release from Gel-Cip for bacterial inactivation [19]. 
To prolong the release time of antibiotics, some interactions between hydrogel networks and drug were proposed. For example, hydrogel networks containing anionic moieties could interact with small molecule cationic antibiotics via electrostatic attraction and prolong the release of drugs. Libera et al. reported that introduction of Poly (Acrylic Acid) (PAA) into poly (Ethylene Glycol) (PEG) networks to form semi-interpenetrating network structure enabled the hydrogel to load about 40 times more antibiotics (such as amikacin and colistin) than comparable pure PEG hydrogels. Subsequent soaking in physiological buffer released the loaded antibiotics for periods as long as 30 days [20] Yang and Hedrick et al. fabricated biodegradable polycarbonate-based ABA tri-block copolyelectrolyte hydrogels and found that cationic trimethylammonium containing hydrogels and anionic carboxylic acid functionalized hydrogels can sustain the release of negative charged (diclofenac) and positively charged (vancomycin) therapeutics, respectively [21]. Alvarez-Lorenzo et al. developed hydrogels based on cyclodextrin and agar which could interact with drugs by formation of inclusion complexes and/or ionic bonds [22]. Furthermore, some composite hydrogels containing nanoparticles or micelles that interacted and loaded with drugs are powerful drug delivery system, the unique architecture of hydrogels benefited the prolonged release of drugs. For example, a system combining micelle drug delivery with supramolecular hydrogel assemblies based on cholesterol-containing ABA tri-block copolymers was prepared, which represented an elegant marriage of aqueous hydrophobic drug delivery and next generation injectable viscoelastic materials. The antimicrobial composite hydrogels demonstrated strong microbicidal activity against Gram-negative and Gram-positive bacteria, and C. albicans fungus. Furthermore, amphotericin B-loaded micelles embedded within the hydrogel demonstrated that the release of drug sustained over 4 days and exhibited effective eradication of fungi [23] Shukla et al. also developed highly tunable, antibacterial hydrogels based on gellan gum, $\mathrm{CaCl}_{2}$, vancomycin, and vancomycin loaded graphitized Carbon Black Nanoparticles (CNPs). Therapeutically effective concentrations of vancomycin were released over 6 to 9 days for nonCNP and composite hydrogels, respectively. The phenomenon of slow vancomycin release from the CNPs hydrogels maybe caused by intermolecular interactions between gellan and vancomycin [24].

Unlike physical incorporation of antibiotics into hydrogel networks, Forsythe et al. prepared a click cross-linked hydrogel by conjugating ciprofloxacin through a photo-cleavable linker to the hydrogel network structure. Native ciprofloxacin could be released into the surrounding environment when the hydrogel material was irradiated under $365 \mathrm{~nm}$ UV light at low intensity [25] Wu et al. reported that Gentamycin Sulfate (GS)-loaded carboxymethylchitosan hydrogels that cross-linked by genipin not only showed superb inhibition on bacteria growth and biofilm formation of S. aureus but also significantly enhanced the adhesion, proliferation, and differentiation of MC3T3-E1 cells. The dual functions were attributed to the positive charged chitosan-based hydrogel could selectively disrupt the wall/membrane of bacteria and promote cell adhesion and proliferation, while the loading of GS improved the antibacterial efficiency and was beneficial for the osteoblastic cell response [26].

\section{Hydrogels Loaded with Silver Nanoparticles or Silver Ions}

The use of silver as an antimicrobial dates back century, when solutions of silver salt were commonly consumed for the treatment of illness. Although the mechanism of antimicrobial action was still not completely understood and they could cause apoptosis and necrosis of mammalian cells, Ag nanoparticles had potential use in biomedical application given their known antimicrobial properties against a broad range of bacteria and fungi [7]. One of accepted mechanism for the antimicrobial activity of silver was that the silver ions caused the cell membrane disruption and subsequent lysis.1 Due to the properties of silver nanoparticles depended on their size, shape, and composition as well as on their spatial arrangement, preparing stable silver nanoparticles with defined dimension and controlled spatial architecture was an important goal that remained a significant challenge [27]. Therefore, a number of hydrogels have been recently used for the assembly and growth of silver nanoparticles. Polymers employed for construction of scaffolds for silver nanoparticles not only included synthetic polymers such as poly(acrylamide-co-acrylic acid) [28], poly(N-vinyl pyrrolidone) (PVP), [29] PAA, [30] PEG, [31] poly(vinyl alcohol) (PVA), [32] polyacrylamide (PAM), [33,34] and PNIPAAm, [35,36] but also included natural polymers such as dextran, [37] chitosan, [38] cellulose [39,40] and protein [41] Polymeric hydrogels acted as carriers for the loading of silver could modulate their toxicity in the practical application.

Despite composite hydrogels containing silver can be fabricated by directly adding silver ions, $[42,43]$ or silver nanoparticles into hydrogel $[44,45]$ the most common strategy was in situ synthesis of silver nanoparticles, where the silver ions were controllably reduced to be silver nanoparticles in the hydrogel networks. The reducing agents were employed to in situ synthesis of silver nanoparticles, including sodium borohydride $[46,47]$ sodium hypophosphite, [48] $\mathrm{N}_{2} \mathrm{H}_{4}$, [43] citrate, [49] and Vitamin C, [50] as well as bioactive molecules from plant leaf [41,51]. Silver nanoparticles can also be synthesized by reduction of silver ions with the functional moieties of hydrogel networks including carbonyl groups, [26,52] catechol groups, [53] and poly(ferrocenylsilane) [36] without using any additive reducing agents. Moreover, silver nanoparticles could be obtained by radiation and hydrothermal treatment [6,41,54-57]. Both chemical modification and physical assembly were applied to construct composite hydrogels with special structure and many strategies were applied to controllably load sliver into hydrogel networks.

For example, $\mathrm{Wu}$ et al. synthesized thermoplastic hydrogel based on multi-block PEG-polyhedral oligosilsesquioxane (POSS) polyurethanes and electrospun into nanofibrous webs with $\mathrm{AgNO}_{3}$. 
The silver-containing nanofibrous hydrogel featured outstanding biofilm resistance, with biofilm formation taking hold only after 14 days of incubation in daily refreshed bacterial cultures [42]. Werner et al. designed multilayer hydrogel based on PEG and heparin for prolonged antimicrobial activity. The silver free star-PEG-heparin layer on the top of an Ag nanoparticles containing star-PEG-heparin layer resulted in a hemocompatible materials with durable antimicrobial functionality [58]. The works employed polymeric hydrogels as template for the synthesis of Ag nanoparticles were also widely reported in other literatures [46] Ozdogan et al. prepared carboxymethyl cellulose-Ag nanoparticle hydrogels by chemical cross-linking with fumaric acid and coated them on cotton fabric. They found that the cotton fabric maintained stable antimicrobial properties after washing process [59] Bajipai et al. prepared Ag nanoparticle-loaded poly (acrylamide-co-N-vinyl-2-pyrolidone) hydrogels by breathing-in/breathing-out (BI-BO) approach. As the number of BI-BO cycles increased, both the amount of the silver nanoparticles loaded into this hydrogel and the antibacterial activity increased. The sample underwent three BI-BO cycles showed optimal bacterial activity [60].

One of the main applications of silver loaded hydrogel is wound dressing, due to the combination of ability for keeping moist environment of hydrogels and the antimicrobial property of silver. For example, Boonkaew et al. developed Ag nanoparticle-loaded poly (2-acrylamido-2-methylpropane sulfonic acid) by gamma irradiation for burn wound dressing. The hydrogel could decrease body fluid loss and maintain moist environment for wound, and the cumulative release of silver from the hydrogel was about $80 \%$ at 3 days [56]. Chang and Zhang et al. reported regenerated cellulose/silver nanoparticle hydrogel in $\mathrm{NaOH} /$ urea aqueous system via hydrothermal method. The hydrogel exhibited good mechanical properties and biocompatibility, as well as remarkable bacteriostatic ability against S. aureus and E. coli. The in vivo animal experiment indicated that the average time for wound healing of composite hydrogel group was nearly 3 days in advance compared to that of gauze group. The mechanism of cellulose/ silver nanoparticle hydrogels to promote the infected wound healing is shown in (Figure 2). The porous architecture of hydrogel provided enough channels for sufficient air permeability and exudate absorption, while good swelling ration maintained a moist environment over wound and resulted in excellent biocompatibility. Especially, Ag nanoparticles embraced in the hydrogel networks enhanced antibacterial activities in the infected wound healing process.6 Recently, Hasan et al. reported Ag nanoparticle which was obtained by reducing silver nitrate with chitosan and Poly Ethylene Glycol (PEG) solution impregnated chitosan-PEG hydrogel was synthesized by cross-linked using glutaraldehyde. The resulted hydrogels showed a slow and sustained release of Ag nanoparticles over a period of at least 7 days, and enhanced antimicrobial and antioxidant properties in vitro as well as improved wound healing capability in vivo in diabetic rabbits [61].

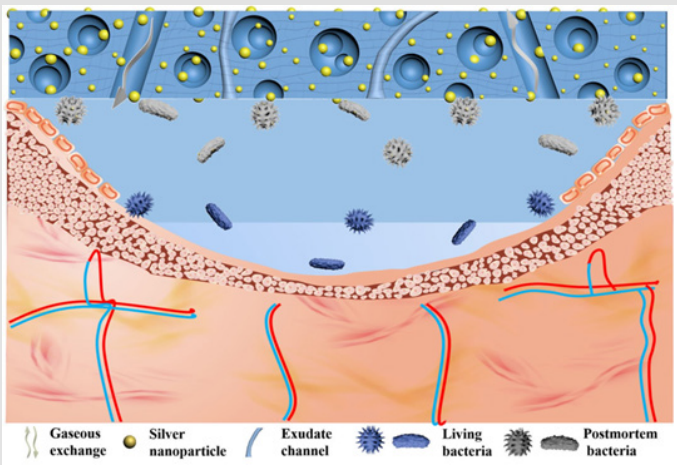

Figure 2: The possible mechanism of the cellulose/ silver nanoparticle sponge to promote the infected wounds healing: the abundant 3D porous structure of the cellulose sponge provided the channels for sufficient air permeability and exudate absorption, and the silver in turn enhanced antibacterial activities, leading to the excellent infected-wounds healing effect [6].

\section{Hydrogels Loaded with Other Metals or Their Compounds}

In addition to the silver nanoparticles, other metals, such as copper, [62] zinc, [63] cobalt, [64] cerium, [65] and aurum,66 or their compounds were also found to have antibacterial properties against both Gram-positive and Gram-negative bacteria and proposed to be incorporated into antimicrobial hydrogels. Villanueva and Copello et al. prepared starch-based hydrogel reinforced with silicacoated copper nanoparticles. The hydrogels were demonstrated to maintain antimicrobial activity against E. coli and S. aures for at least four cycles of use and showed good biocompatibility [66,67]. Carboxymethyl cellulose/CuO nanocomposite hydrogels were synthesized through the in-situ formation of $\mathrm{CuO}$ nanoparticles within swollen carboxymethyl hydrogels. The size of $\mathrm{CuO}$ nanoparticles were 40 75 $\mathrm{nm}$ within hydrogel matrix, and the number of nanoparticles increased at higher concentration of $\mathrm{Cu}^{2+}$. The composite hydrogels showed very good antibacterial activity against both Gram-positive and Gram-negative bactericides [68]. Similarly, Chu et al. developed a series of carboxymethyl chitosan/ $\mathrm{CuO}$ nanocomposite hydrogels with the size of $\mathrm{CuO}$ nanoparticles from $20 \mathrm{~nm}$ to $50 \mathrm{~nm}$ by oxidation of copper ions in the presence of sodium hydroxide. The nanocomposite hydrogel showed excellent antibacterial behavior against Staphylococcus aureus and Escherichia coli [69]. In addition, ZnO impregnated mesoporous silica (MCM-41) were incorporated into the carboxymethyl cellulose (CMC) hydrogel with citric acid as cross linker, where the CMC/ZnO-MCM-41 also exhibited good antimicrobial activity [70]. Zinc-alginate hydrogel prepared by internal setting method by using $\mathrm{ZnCO}_{3}$ and D-Gluconic Acid- $\delta$--Lactone (GDL). Internal gelation can be mediated by zinc ions to obtain hydrogels with high structural homogeneity, high water content, and antimicrobial activity against E. coli [71]. The hydrogel composed by alginate, hyaluronate, and cerium ions showed effective bioactive and antimicrobial ability, allowed the granules injection, and enhanced the osteoconductive ability of the substitute [70]. 
Liposomes have attracted significant attention as a class of antimicrobial delivery vehicles owing to their unique features including biocompatible lip materials, a bilayer structure capable of fusing with microbial membrane, readily modifiable formation properties, and high drug loading capacity. Zhang et al. [72] developed a hydrogel formation that contained $\mathrm{pH}$ responsive gold nanoparticle-stabilized liposomes for topical antimicrobial activity. The hydrogel not only preserved the structural integrity of the nanoparticle stabilized liposomes, but also allowed for controllable viscoelasticity and tunable liposome release rate. As shown in (Figure 3), only nucleoids with blue were seen for untreated bacterial (A), whereas weak sporadic red dots in the peripheral area of the bacterial nucleoid after incubating with AuC-liposome hydrogel at $\mathrm{pH}=7.4$ for $30 \mathrm{~min}$ can be observed (B). However, a significant increase of RhB fluorescence signal around the bacterial nucleoid appeared after the bacteria were incubated with AuCliposome hydrogel at $\mathrm{pH}=4.5$ (C). The zoomed-in image showed that RhB signal was exclusively and evenly distributed around the bacterial nucleoid, consistent with the fusion between liposomes and the bacterial membrane (D). The differential liposome-bacteria fusion activity clearly demonstrated that the AuC-lipsome hydrogel can readily release AuC-liposome to the bacteria culture (E), which then fuse with bacterial membrane. When topically applied onto mouse skin, the hydrogel did not generate any observable skin toxicity within a 7-day treatment. The hydrogel containing nanoparticle-stabilized liposomes hold great promise for topical applications against various microbial infections [66].

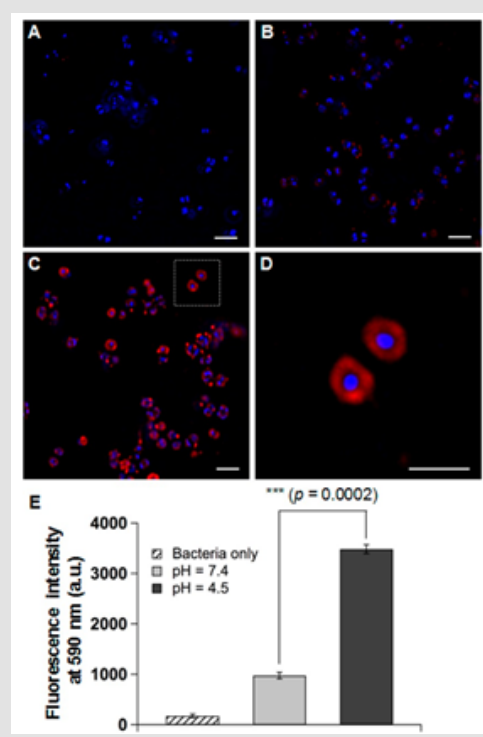

Figure 3: Fluorescence study of the fusion interaction between hydrogel-released liposomes and S. aureus bacteria. Liposomes were labeled with fluorescent dye $\mathrm{RhB}$ (red) and the bacteria were stained with DAPI (blue). (A) Control bacteria without any treatment, (B) bacteria incubated with AuC-liposome hydrogel (PEGDMA $0.8 \mathrm{vol} \%)$ at $\mathrm{pH}=7.4$, (C) bacteria incubated with AuClipsome hydrogel (PEGDMA 0.8 vol\%) at $\mathrm{pH}=4.5$, (D) a zoomed-in image of $(\mathrm{C})$, and (E) overall fluorescence intensity comparison of the bacterial sample of (A-C). The scale bars in (A-D) represent $1 \mu \mathrm{m}$. [66].

\section{Hydrogels Loaded with Other Compounds}

Other compounds with antimicrobial activity were also incorporated into hydrogel matrix for the construction of composite hydrogels. For example, trisodium phosphate and acidified sodium chloride was used as antimicrobials to incorporate into starch and alginate-based hydrogel, respectively [73]. Starch/polyvinyl alcohol hydrogels containing boron complexes showed good antimicrobial activity with $14 \mathrm{~mm}$ inhibition zones against E. coli which was comparable with the standard antibiotic erythromycin [74]. Photosensitizers meso-Tetra (N-methyl-4-pyridyl) Porphine Tetra Tosylate (TMP) and Methylene Blue (MB) were loaded into crosslinked poly (methyl vinyl ether-co-maleic acid) for preparation of composite hydrogels. The released TMP or MB could completely kill clinical strains of methicillin-resistant $\mathrm{S}$. aureus and B. cepacian [75]. 5,6-dimethoxy-1-indanone N4-allyl thiosemicarbazone (TSC) employed as antimicrobial agent was incorporated into PHEMAco- $\beta$-cyclodextrin hydrogel and cross-linked hydroxypropyl- $\beta$ cyclodextrin hydrogel, respectively. Both systems enabled the loading of high TSC cargos and provided sustained release for at least two weeks, which inhibited the growth of two prominent bacteria associated with ocular infections [21] Hughes et al. reported that seaweed extract contained PVA/PVP hydrogel was active against nine clinical relevant wound pathogens. The dressing was effective in inhibiting $70-90 \%$ of the bacterial population within the first $30 \mathrm{~min}$ and sustained released up to $97 \mathrm{~h}$, which antimicrobial activity was comparable to the commercial silver dressings [76]. Furthermore, cetylpyridinium chloride and benzalkonium bromide were also used as antimicrobial agents for preparation of composite hydrogels with antibacterial activity $[77,78]$ Jung et al. reported the preparation of chemically cross-linked cyclosophoraose (Cys)/ cellulose hydrogel for the efficient delivery of galangin. The result indicated that Cys/cellulose hydrogel outperformed the cellulose and $\mathrm{CD} /$ cellulose hydrogels as a galangin delivery system in terms of loading amount and sustainable release, where galangin encapsulated into the Cys/cellulose hydrogel network allowed sustained and robust release for $48 \mathrm{~h}$, and the hydrogel exhibited excellent antibacterial activity after long incubation period (72h) with S. aureus [79].

Besides the small molecules mentioned above, the delivery of some macromolecular antimicrobials by using hydrogels have also been largely understudied, due to many medical devices such as contact lenses, intervascular catheters and prosthetic implants utilized hydrogel materials that succumb to microbial attachment or biofilm formation [80]. Antimicrobial Peptides (AMPs) are highly advantageous over the conventional antibiotics because of their broad-spectrum activity, selectivity, and minimal bacterial resistance observed so far [81] Liskamp et al. described a single step immobilization-polymerization strategy of a highly active antimicrobial peptide into a soft hydrogel network on a PET surface using thiol-ene chemistry. (Figure 4) shows the Coomassie brilliant blue stained hydrogel for visualization of the distribution of the immobilized peptide with the hydrogel and their 
antibacterial activity. With increasing the concentration of peptide in the hydrogel networks, their antibacterial activities increased and complete killing of the inoculum was observed for 6,8 , and $10 \mathrm{wt}$. \% hydrogels. The composite hydrogels incorporating gelatin methacryloyl (GelMA) and methacryloyl-substituted recombinant human tropoelastin (MeTro) via visible light-induced crosslinking with conjugation of antimicrobial peptide Tet213 to the hydrogels were developed. The engineered MeTro/GelMA-Tet213 hydrogels showed potent bactericidal activity against Gram (+) and (-) bacteria and prevented infection and promoted healing of chronic wounds [82]. An in situ forming biodegradable hydrogel composed of biodegradable poly (ethylene glycol) maleate citrate and poly (ethylene glycol) diacrylate was developed, and it showed and promoted wound healing after conjugation with antimicrobial peptides [83]. A novel self-assembling hydrogel of RADA16 has been prepared for Antimicrobial Peptides (AMPs) delivery. The release study demonstrated that the sustained release of AMPs could be obtained until 28 days. Importantly, the hydrogel has an inhibitory effect on the proliferation of S. aureus and enhances osteogenesis [84]. Moreover, unlike these hydrogels containing AMPs, AMPs as backbone for fabrication of antimicrobial hydrogels will be discussed in the section 3.3 .

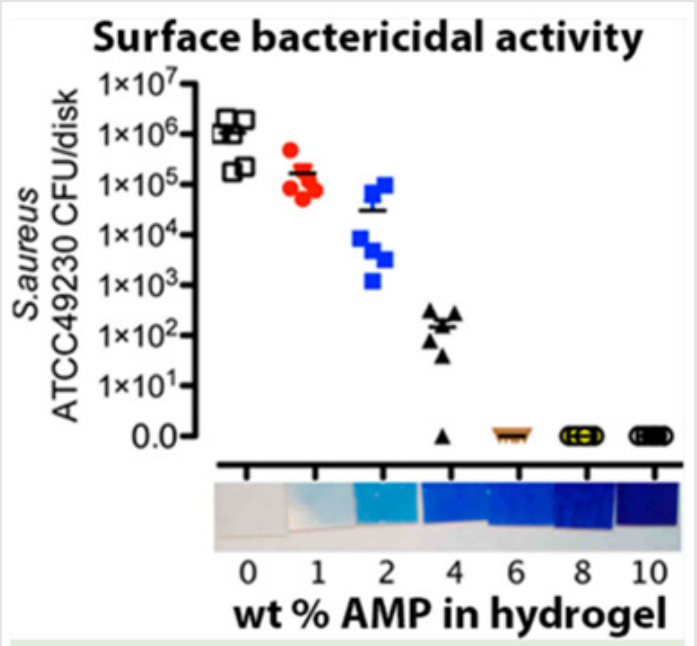

Figure 4: Surface bactericidal activity and Coomassie brilliant blue staining of hydrogel networks [80].

\section{Hydrogels with Inherent Antimicrobial Activity}

Although various antimicrobial agents can be encapsulated into hydrogel networks which only acted as structural materials to achieve antimicrobial properties of hydrogels, some limitations in their real application must be considered. On one hand, a small amount of the antimicrobial agents released from hydrogel matrix cannot kill microbes, while too much antimicrobial agents usually induced cytotoxicity of materials in clinic application. So, the control of release rate for antimicrobial agents is important for antimicrobial properties of materials. On the other hand, antimicrobial agents must be preloaded into hydrogels networks through effective interaction between agents and networks and only partial agents can be successfully encapsulated in the hydrogels. Therefore, a kind of hydrogels with inherent antimicrobial network were developed, including natural polymer-based hydrogels, synthetic polymerbased hydrogel, peptide hydrogel, and hydrogel from the mixture of them. The common feature of inherent antimicrobial hydrogel is the presence of cationic moieties in the hydrogel networks.

\section{Natural Polymer-Based Hydrogels}

Chitosan is a cationic polysaccharide, with properties of nontoxic, biocompatible and biodegradable, whose advantages provide widely applications in biomedicine [85] food, [86] metal ion adsorption, $[87,88]$ and textile [89]. Importantly, chitosan is an inherently antimicrobial material attributed to its positive charges, which could improve the interaction with the negatively charged bacteria cell surface and destroy bacterial cytoplasmic membranes to lead to death [90]. Antibacterial hydrogels based on chitosan can be prepared by directly chemical cross-linking of chitosan with different cross-linkers. For example, terephthaloyl diisothiocyanate was employed as cross-linker for the fabrication of chitosan hydrogels. The resulting hydrogels showed stronger activity against Gram-positive bacteria than against Gram-negative bacteria and showed significant inhibitory effect on the fungi. Furthermore, the antimicrobial activity of hydrogels improved by increasing the degree of cross-linking [91].

Similar results were also observed for the multi-walled carbon nanotubes modified chitosan hydrogels by chemical cross-linking with trimellitic anhydride isothiocyanate [92] oxalyl bis 4-(2,5-dioxo-2H-pyrrol-1(5H)-yl) benzamide cross-linked chitosan hydrogel, and N',N'-bis [4-(isothiocyanate carbonyl) phenyl] pyromellitimide cross-linked chitosan hydrogel [93] Shi et al. reported that the hydrogel prepared by electrophoretic co-deposition of chitosan and alkynyl chitosan exhibited better antibacterial activities than pure chitosan hydrogel because the hydrophobic properties of alkynyl chitosan facilitated the integration between bacteria cell and chitosan derivative chains [94]. Antibacterial and conductive injectable hydrogels were fabricated by cross-linking Quaternized Chitosan-G-Polyaniline (QCSP) and benzaldehyde group functionalized poly (ethylene glycol)-co-poly (glycerol sebacate) (PEGS-FA). These hydrogels presented good electroactivity, antibacterial activity, adhesiveness, conductivity, swelling ratio, and biocompatibility. The introduction of polyaniline onto quaternized chitosan copolymer decreased the cytotoxicity of hydrogels and enhanced their antibacterial activity by a synergetic effect. Furthermore, the electro-active hydrogel showed good antibacterial activity, free radical scavenging capacity, and self-healing [95].

Novel multi-network hydrogels with good antimicrobial and cell anti-adhesive properties were synthesized by using chitosan as an antimicrobial constituent (the first network), and zwitterionic sulfopropylbetaine (PDMAPS) as an cell anti-adhesive constituent (the second network) via chemical and photochemical 
crosslinking methods. The tough hydrogels had highly compressive strength, tensile strength and antimicrobial activities [96] Chan-Park et al. reported an antimicrobial hydrogel based on dimethyldecylammonium chitosan-graft-poly (ethylene glycol) methacrylate (DMDC-Q-g-EM) and ploy(ethylene glycol) diacrylate, which had excellent antimicrobial efficacy against P. aeruginosa, E. coli, S. aureus, and F. solani. The high antifungal activity of hydrogels may be due to the compatibility of the chitosan derivatives with fungal cell wall, which contains a significant amount of chitin, enabling close approach of the hydrogel to the cell membrane (Figure 5a). Even after three challenges with high concentration of microbes, the dead cells easily washed from the hydrogel and the antimicrobial activity of hydrogels was well preserved in the fourth challenge, as shown in (Figure $5 \mathrm{~b}$ ). Figures $5 \mathrm{c}$-5h shows the morphologies of various microbes on DMDC-Q-g-EM hydrogels and control, indicating the excellent their antimicrobial activities [97].

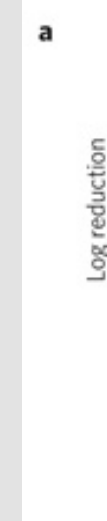

b
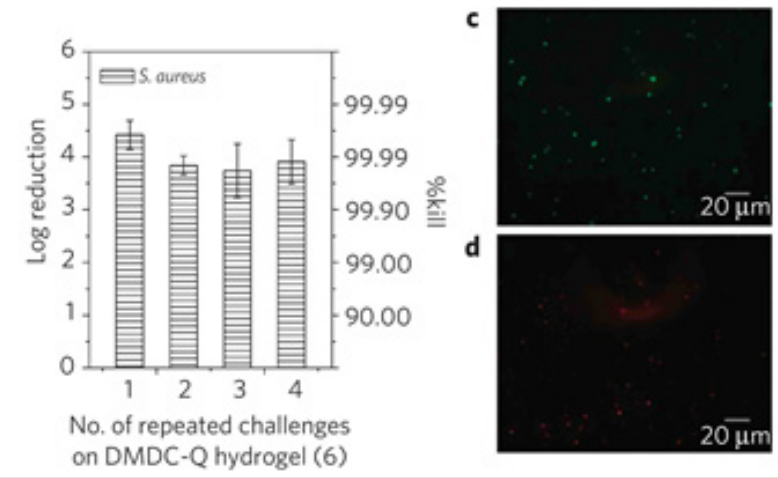

e
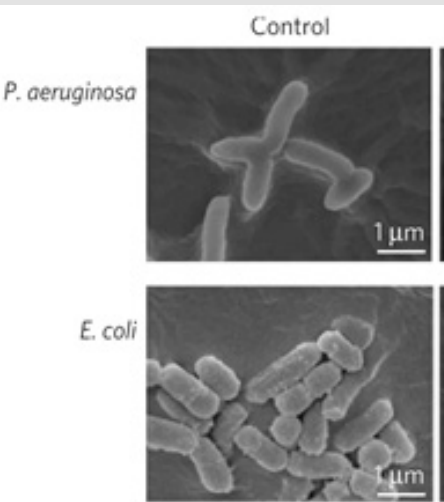

g
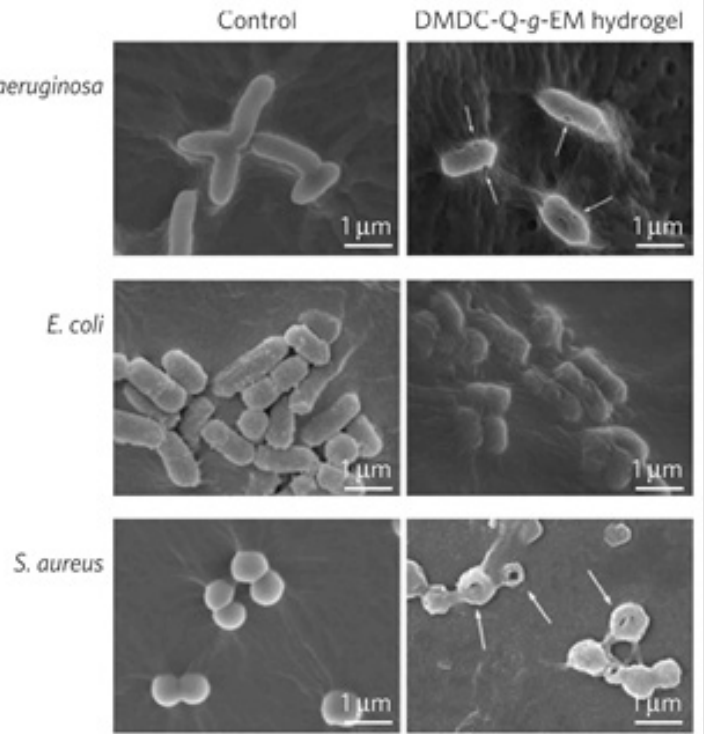

h

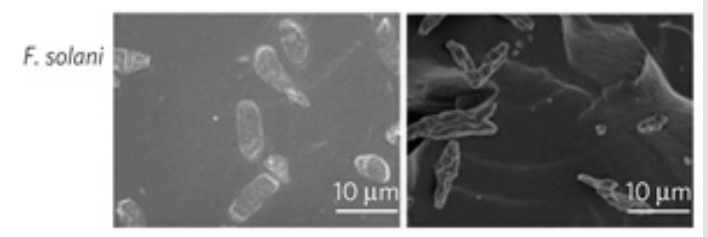

Figure 5: Antimicrobial activities of quaternized ammonium chitosan-graft-poly (ethylene glycol) methacrylate (qC-g-EM) hydrogels against various bacteria and fungi. a, Log reduction and \%Kill of four pathogens on various qC-g-EM hydrogels. b, Multi-use antimicrobial activities of DMDC-Q-g-EM hydrogel against S. aureus. Error bar represent mean \pm standard deviation of mean for $n=3$. c, d, Live/dead bacteria viability of S. aureus on PEG13DA control (c) and DMDC-Q-g-EM hydrogel (d) after $1 \mathrm{~h}$ incubation at $37^{\circ} \mathrm{C}$ and washing (scale bar $=20 \mu \mathrm{m}$ ).e-h, Morphology of various pathogens in contact with DNDC-Q-g-EM hydrogel (right) and control (left). Arrows indicate lesions and holes on the cell membrane after contact with DMDC-g-EM hydrogel [97].

Moreover, other natural polymers also can be used to develop hydrogel with antimicrobial activity after chemical modification. For example, biocompatible cellulose-based superabsorbent hydrogels were prepared by introduction of quaternary ammonium groups into hydrogel networks. These hydrogels exhibited good mechanical performances, superabsorbent properties, biocompatibilities, and antimicrobial activities against $S$. cerevisiae [98]. Compared to synthetic hydrogels, natural polymeric hydrogels usually had good biocompatibility due to their origin. However, natural polymer-based hydrogels with antimicrobial activity were seldom reported, except chitosan. So, more attentions may be paid to natural polymers for the construction of antimicrobial hydrogels for biomedical application due to their advantageous properties.

\section{Synthetic Polymer Based Hydrogels}

PEG is an FDA approved hydrophilic polymer possessing excellent biocompatibility, which has been widely applied in biomedical fields [99]. Therefore, antimicrobial PEG-based hydrogels could be constructed by grafting Quaternary Ammonium Salts (QAs) as antimicrobial moieties onto PEG hydrogel backbones. For example, Zhu et al. reported that fully biodegradable antimicrobial hydrogel was prepared via thiol-ene click reaction under human physiological conditions by using multifunctional PEG derivatives as precursors and dodecyl bis(2-hdyroxyethyl) methyammonium chloride as a co-monomer. The hydrogels with fewer QAs moieties possessed both strong antibacterial 
abilities and low toxicity [100]. They also fabricated ultrathin hydrogel films via layer-by-layer technique and click chemistry by using multifunctional PEG derivatives as precursors. Owing to the presence of quaternary ammonium groups in the hydrogel backbone, these hydrogels show significant antibacterial activity against both Gram-negative and Gram-positive bacteria, enhanced by the increasing in hydrogel thickness [99] Fu et al. synthesized antibacterial PEG-based hydrogel by combination of Epoxy Amine (QA) and thiol-ene click reaction.

The structure of four types hydrogels (Pendant QA/3-arm thiol gel, Pendant QA/4-arm thiol gel, Chian-End QA/3-arm thiol gel, and Chian-End $\mathrm{QA} / 4$-arm thiol gel) were well investigated, and their mechanical properties could be tuned by varying the architecture of the networks. These hydrogels containing QAs exhibited excellent antibacterial activity against S. aureus [101]. Recently, antibacterial hydrogels fabricated from an amine functional linear-dendritic hybrid based on linear poly(ethylene glycol) and dendritic 2,2-bis(hydroxymethyl) propionic acid, with a di-N-hydroxysuccinimide functional poly(ethylene glycol) as cross linker showed broad spectrum antibacterial effect both in vitro toward a range of relevant bacterial strains and in an in vivo mouse model of surgical site infection [102]. PVP has nontoxicity and good biocompatibility and has been widely applied for wound dressing. Many works focused on the development of hydrogels by blending PVP and other biocompatible polymers, which will be discussed in the section of other polymeric hydrogels. Zheng and Guan et al. reported antimicrobial PVP-GP hydorgels obtained by polycondensation of hexamethylenediamine and guanidine hydrochloride, followed by cross-linking reaction using $\mathrm{N}$-vinyl pyrrolidone and N, N'-methylene bisacrylamide. Kirby-Bauer inhibition zone test and microbe penetration assay proved that PVP-GP hydrogel had high antimicrobial activity [103].

Hydrogel with semi-interpenetrating networks were prepared by free radical cross-linking copolymerization of HEMA and itaconic acid, in the presence of PVP. The resulting hydrogel exhibited effective antibacterial ability against $C$. albicans, rather than against $S$. aureus and E. coli [104]. Moreover, injectable hydrogels consisted of enzymatic cross-linking of epsilon-poly-L-lysine (EPL) backbone and phenol groups of hydroxyphenylpropionic acid (HPA) were fabricated for wound infection prevention. These hydrogels exhibited adjustable mechanical performance, good adhesive strength, and excellent biocompatible and inherently antibacterial properties [105]. Poly (tannic acid) hydrogel prepared by crosslinking tannic acid molecules with an epoxy cross-linker were biocompatible and biodegradable, which was applied in wound healing. Hydrogel was completely degraded at pH9 hydrolytically in 9 days, and the degraded hydrogel displayed strong antimicrobial behavior against Gram positive S. aureus, B. Subtilis, Gram negative P. aeruginosa bacteria strains and C. albicans fungus strain [106] Hedrick and Yang et al. reported an antimicrobial hydrogel based on vitamin E-containing polycarbonate copolymers synthesized by organo-catalytic ring-opening polymerization.

The hydrogel was composed by ABA type tri-block copolymer consisting of a hydrophilic PEG middle block flanked on both blocks, and biocidal cationic polycarbonates possessing Vitamin E moieties. The incorporation of Vitamin $\mathrm{E}$ in the cationic polycarbonates enhanced the antimicrobial efficacy as a result of increasing of hydrophobicity of the polymers. These hydrogels exhibited thixotropic properties, and effective against both Gram-positive and Gram-negative bacteria as well as fungus with more than $99.9 \%$ killing efficiency upon contact. Importantly, the hydrogels containing the cationic polycarbonates and/or fluconazole at their minimum biocidal concentrations do not induced significant cytotoxicity towards human dermal fibroblasts [107]. Antimicrobial polycarbonate hydrogels were synthesized from tertiary amine-functionalized eight-membered cyclic carbonate monomers. Due to the cross-linked polycarbonates were quternized with methyl iodide, hydrogels showed broadspectrum antimicrobial ability and rapid biodegradability on order of $4 \sim 6$ days where the degradation products were nontoxic to mammalian cells [108]. Lately, a novel photoresponsive poly[2((4,5-dimethoxy-2-nitrobenzyl) oxy)-N-(2-(methacryloyloxy) ethyl)-N,N-dimethyl-2-oxoethan-1-aminium] (polyCBNA) hydrogel consisted of photolabile 4,5-dimethoxy-2-nitrobenzyl and cationic quaternary ammonium groups was developed. The photolabile groups enabled polyCBNA hydrogels to be finely switched from cationic to zwitterionic form by UV irradiation. The positive surface charge of hydrogel were demonstrated to kill bacteria effectively, and then tuned to zwitterionic form after photolysis, which caused desorption of their surfaces attached protein and resist further bacterial attachment (Figure 6) [109].

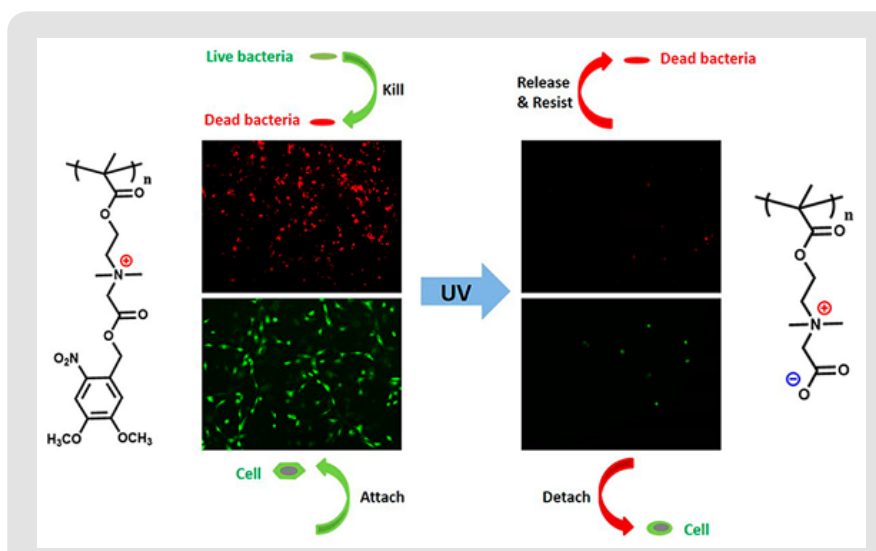

Figure 6: The chemical structure, and schematic bactericidal and bacteria-resistant functions of the novel light-responsive hydrogels [109].

\section{Peptide Based Hydrogels}

In addition to natural and synthetic polymer-based hydrogels, several peptide-containing antimicrobial hydrogels have also been 
reported. For instance, Schneider et al. designed hydrogel scaffold from the self-assembling peptide, MAX1, for tissue regeneration whose surface exhibited inherent antibacterial activity. Hydrogel could cause the inner and outer membrane disruption leading to cell death upon cellular contact with the gel surface. Coculture experiments showed that, when NIH3T3 fibroblasts and a mixture of A. xylosoxidans and S. maltophilia were introduced onto the hydrogel, its surface inhibited bacterial proliferation yet allowed mammalian cell adhesion and proliferation, indicating that the surfaces are selective [110]. They also developed MARG1 containing 20-residue peptide which designed to undergo a triggered intramolecular folding event, adopting an amphiphilic $\beta$ hairpin that is prone to self-assembly. This injectable $\beta$-hairpin peptide hydrogel could effectively kill methicillin-resistant S. aureus [111]. Roy and Das developed antimicrobial hydrogels by using L-tryptophon based cationic amphiphilic hydrogelators with varying degree of hydrophobicity, whose bactericidal effect of cationic hydrogelators comparable or better than that of clinically available antibiotics. These hydrogel could selectively attack the bacterial pathogens while remained biocompatibility to the mammalian cells [112]. Malhotra et al. reported $\alpha / \gamma$ hybrid peptides of Boc-D-Phe- $\gamma 4$-L-Phe-PEA (NH007) and Boc-L-Phe- $\gamma 4$ L-Phe-PEA (NH009) to improve the proteolytic stability. Both of dipeptides could self-assemble into gels in DMSO (3\%-5\% w/v). Both gels exhibited broad spectrum antibacterial activity against Escherichia coli, Bacillus subtilis, Pseudomonas aeruginosa, and Staphylococcus aureus. The antibacterial capability of NH009 gels was a little higher than NH007, which maybe attribute to the better interactions of L-L dipeptide with bacterial membrane. In addition, NH009 gels were more effective against Pseudomonas aeruginosa due to resistance. The underlying mechanism for antibacterial activities of gels proven to be entrapment in the gel network and followed by adhesion and membrane rupture [113].

The self-assembly of a sparingly soluble antibiotic (ciprofloxacin) and a hydrophobic tri-peptide (DLeu-Phe-Phe) into supramolecular nanostructure that yield a macroscopic hydrogel at physiological pH. The ciprofloxacin-peptide assembled hydrogel, which formed by non-covalent interactions and retained the activity of ciprofloxacin over a prolonged release timescale, was evaluated against S. aureus, E. coli, and K. pneumoniae. While toxic to bacteria, no major cytotoxicity was seen in haemolysis assays of human red blood cells or in mouse fibroblast cell cultures. This approach of drug incorporation into the nanostructure of a simple tri-peptide hydrogel by self-assembly may have important applications for cost-effective wound dressings and novel antimicrobial formulations [114]. Taking the advantage of the intermolecular $\pi-\pi$ stacking interactions of Fmoc and phenyl group, supramolecular hydrogels were developed via a co-assembly mechanism by combining the Fmoc-F hydrogelator with another commercial Fmoc-L as antimicrobial building block. These amino acid based hydrogels exhibited selective Gram-positive bactericidal activity (Figure 7), where the appearance of S. aureus converted from clear edges and smooth bodies to cellular deformation and surface collapse after contact with hydrogel for $2 \mathrm{~h}$ and $\mathrm{S}$. aureus bacteria were stained red after the co-assembled hydrogel confirmed the disruption of the bacterial cell wall and membrane since red fluorescent propidium iodide could penetrate only bacteria with destroyed cell walls and membranes. This work provided a novel strategy to fabricate antibacterial hydrogel easily and cost-effectively without involving complicated organic synthesis and purification processes, as compared to the commonly used antimicrobial hydrogels based on cationic materials [115].
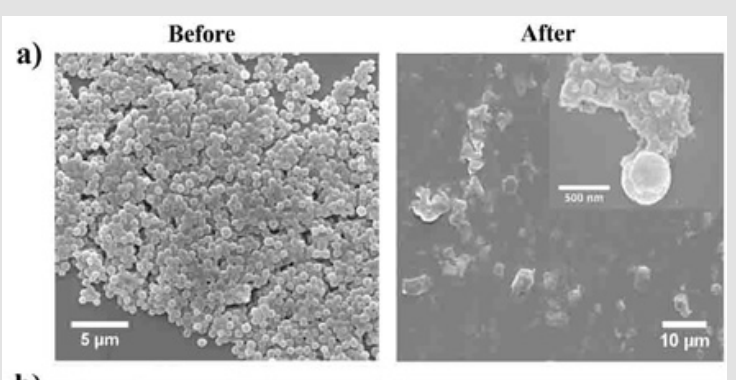

b)
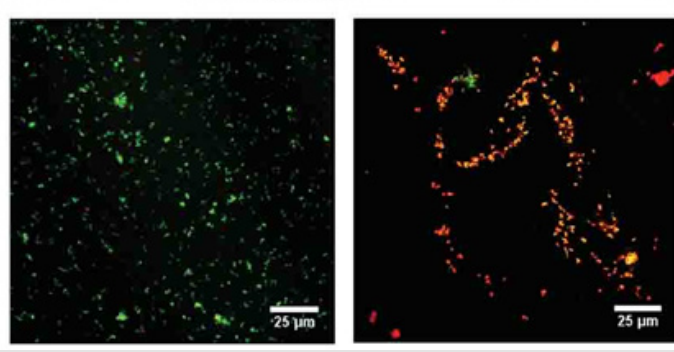

Figure 7:

a) Representative of SEM images and

b) overlapping florescence images for live/dead bacterial staining assay of $\mathrm{S}$. aureus before and after contact with the co-assembled $(\mathrm{F}+\mathrm{L})$ hydrogel for $2 \mathrm{~h}$ [115].

\section{Other Polymeric Hydrogels}

Several antimicrobial hydrogels were developed by combination of natural polymer and synthetic polymers. For example, PVA hydrogels containing different concentrations of chitosan with molecular weight of 471 and $101 \mathrm{kDa}$ were cross-linked by gamma irradiation at a dose of $25 \mathrm{kGy}$. The hydrogels were more effective in inhibiting growth of Gram-positive bacteria than that of Gramnegative ones, and the inhibition zone diameter of antibacterial experiment increased with the content and molecular weight of chitosan in the hydrogels [116]. Fluorescent oligo(phenylene ethynlene) (OPE)-DNA hydrogels have been prepared and used for the controllable biocidal activity driven by DNase, where fluorescent OPEs function as photosensitizers in the regulation if biocidal activity and salmon sperm DNA acts as the frame of the hydrogel, and the DNA remained biocompatible aspects and OPEs retain their optical properties in the gel state. After the hydrogel was hydrolyzed by DNase, the released OPEs showed effective biocidal activity under irradiation, which was far greater than the GEL itself 
[117]. Biodegradable hydrogels were synthesized form sodium alginate, ethylene glycol, and acrylic acid. The hydrogels showed strong antibacterial activity against E. coli and S. aureus, while exhibiting good biocompatibility. Furthermore, the in vivo results indicated that hydrogels had an excellent wound healing capacity than that of standard silver sulfadiazine [118] Recently, Li and Tian et al. have synthesized a kind of non-releasing antimicrobial Poly(ionic liquid)/PVA hydrogel dressing with high-strength via chemical polymerization and physical cross-linking. The hydrogel dressing showed good antimicrobial efficacy against bacteria (E. coli, S. aureus and B. subtilis), fungus (C. albicans), and mold (Asp. niger, Asp. oryzae, and Rhizopus). Furthermore, in a murine model, the poly (ionic liquid)/PVA hydrogel exhibited efficiently accelerated cutaneous wound healing through promoting the reconstruction of intact epidermis [119].

Syringe-injectable bio-adhesive hydrogels with inherent antibacterial properties were prepared by mixing polydetran aldehyde as bio-adhesive component and branched polyethylenimine as antimicrobial component, as shown in Figure 8. The hydrogel can kill both Gram-negative and Gram-positive bacteria, while sparing human erythrocytes. Furthermore, the hydrogel was also effective in a cecal ligation and puncture model, preventing sepsis and significantly improving survival [120]. Hydrogels with similar structure composed by chitosan and poly (ethylene glycol) diacrylate were also prepared in presence of pore-formed agents. The chemical initiation system was found more suitable than the UV initiation system for antibacterial activity, while the enhancement of molecular weight of poly (ethylene glycol) diacrylate could improve the antibacterial activity of hydrogels [121]. Other antimicrobial hydrogels constructed by natural/synthetic polymers included dextran/PAM, [122] guar gum/PAA/polyaniline, [123] quaternized chitosan/ PVA/PEO, [124] carrageenan/PVP, [125] chitosan/PEO, [126] Gelatin/ionically modified bacterial cellulose, [126-128] PVA/gelatin,128 Bacterial cellulose/PAA were also well investigated [129].

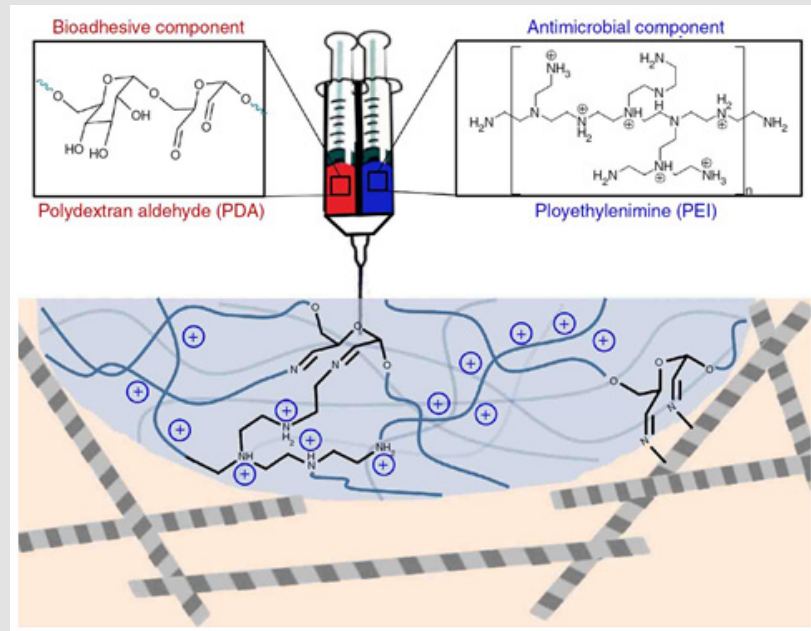

Figure 8: Design of injectable bio-adhesive hydrogels with antibacterial activity [120].

\section{Conclusion}

Owing to microbial infections caused by bacteria and fungi in wound healing and biomedical implant procedure seriously threaten the human health, the development of novel materials with antimicrobial activity is urgently required. Hydrogels as soft materials consisting mostly of water resemble biological soft tissues were considered as ideal wound dressings or implant scaffolds. The recent advances in antimicrobial hydrogels prepared by incorporation of antimicrobial agents into hydrogel networks and cross-linking of inherent antimicrobial polymers were summarized in this mini review. Hydrogels acted as controlled release systems for antibiotics, silver nanoparticles, other metals and their compounds, as well as other compounds were introduced, respectively. Inherent antimicrobial hydrogels composed of natural polymers, synthetic polymers, and peptides as well as their mixture were discussed. Although selected represent works were highlighted, there are still some challenges to be overcome, i.e. antibiotics easily developed drug resistance, the toxicity of metal nanoparticles was difficult to mitigate, and the spectrum width of antimicrobial hydrogels was not clear.

From the clinical application of view, the antimicrobial activity of hydrogels against clinically isolated microbes and multidrug-resistance strains were less evaluated, and the preparation procedure as complicated which will hinder largescale manufacture of materials. For wound dressing application, more attentions were paid on the antibacterial activity and biocompatibility, whereas other properties of the hydrogel, such as mechanical performance, exudate absorption, gas exchange, and maintain moist environments were almost ignored. When hydrogels were applied as antimicrobial implant scaffolds, beside their in vivo biodegradation and biocompatibility, the mechanism of their antimicrobial properties, the toxicity of degradation products, and metabolic products were not systemically investigated. Based on the aforementioned challenges in fundamental research and practical application, the future directions of antimicrobial hydrogels are suggested to be carried out in the following aspects.

First, the antimicrobial activity of hydrogels against clinically isolated microbes and multidrug-resistance strains should be conducted and the mechanism for effective killing microbes and preventing biofilm formation of the hydrogels should be deeply investigated. Second, facile methods for preparation of hydrogels should be developed which will benefit the fabrication cost and large-scale production capability of materials. Third, antimicrobial hydrogels as implant scaffolds are less reported which is also important as wound dressings in clinical application.

\section{Acknowledgement}

This work was financially supported by the National Natural Science Foundation of China (51703174), Hubei Province Science Foundation for Youths (2017CFB154), Startup Foundation of 
Chutian Scholars by Wuhan University of Science and Technology (040288, 040291), and Open Program of Hubei Province of Key Laboratory of Coal Conversion and New Carbon Materials (WKDM201901).

\section{References}

1. Finnegan S, Percival SL (2015) Clinical and Antibiofilm Efficacy of Antimicrobial Hydrogels. Adv Wound care 4: 398-406.

2. Lu Y, Wu Y, Liang J, Libera MR (2015) Self-defensive antibacterial layer-by-layer hydrogel coatings with $\mathrm{pH}$-triggered hydrophobicity. Biomaterials 45: 64-71.

3. Ng VW, Chan JM, Sardon H, Ono RJ, Garcia JM, et al. (2014) Antimicrobial hydrogels: a new weapon in the arsenal against multidrug-resistant infections .Adv Drug Deliver Rev 78: 46-62.

4. Li S, Dong S, Xu W, Tu S, Yan L, et al. (2018) Antibacterial Hydrogels. Adv Sci 5(5): 1700527.

5. Ghobril C, Grinstaff MW(2015) The chemistry and engineering of polymeric hydrogel adhesives for wound closure: a tutorial. Chemi Soc Rev 44: 1820-1835.

6. Ye D, Zhong Z, Xu H, Chang C, Yang Z, et al. (2016) Construction of cellulose/nanosilver sponge materials and their antibacterial activities for infected wounds healing. Cellulose 23: 749-763.

7. Yang K, Han Q Chen B, Zheng Y, Zhang K, et al. (2018) Antimicrobial hydrogels: promising materials for medical application. Int J Nanomed 13: 2217-2263.

8. Hoare TR, Kohane DS (2008) Hydrogels in drug delivery: Progress and challenges. Polymer 49(8): 1993-2007.

9. Hamad B (2010) The antibiotics market.Nat Rev Drug Discov 9(9): 675676.

10. Mc Intyre PW, Wu JL, Kolte R, Zhang R, Gregory Rl, et al. (2019) The antimicrobial properties, cytotoxicity, and differentiation potential of double antibiotic intracanal medicaments loaded into hydrogel system Clin. Oral Invest 23(3): 1051-1059.

11. Hoque J, Bhattacharjee B, Prakash RG, Paramanandham K, Haldar (2017) Dual Function Injectable Hydrogel for Controlled Release of Antibiotic and Local Antibacterial Therapy. Biomacromolecules 19(2): 267-278.

12. Mallick SP, Sagiri SS, Singh VK, Pal K, Pradhan DK, et al. (2014) Biopolymer for medicalApplications. Polym Plast Technol 53: 700-715.

13. Lu M, Liu Y, Huang YC, Huang CJ, Tsai WB(2018) Fabrication of photocrosslinkable glycol chitosan hydrogel as a tissue adhesive. Carbohyd Polym 181: 668-674.

14. De Cicco F, Reverchon E, Adami R, Auriemma G, Russo P, et al. (2014) Carboy. Polym 101: 1216-1224.

15. Zhang L, Wang L, Guo B, Ma PX (2014) Cytocompatible injectable carboxymethyl chitosan/N-isopropylacrylamide hydrogels for localized drug delivery. Carbohyd Polym 103: 110-118.

16. Wang B, Han Y, Lin Q, Liu H, Shen C, et al. (2016) In vitro and in vivo evaluation of xanthan gum-succinic anhydride hydrogels for the ionic strength-sensitive release of antibacterial agents. J Mater Chem B 4: 1853-1861.

17. Lu Z, Zhang J, Yu Z, Liu Q, Liu K (2017) Hydrogel degradation triggered by $\mathrm{pH}$ for the smart release of antibiotics to combat bacterial infection. New J Chem 41(2): 432-436.

18. Anirudhan TS, Mohan Am (2018) Novel pH sensitive dual drug loadedgelatin methacrylate/methacrylic acid hydrogel for the controlled release of antibiotics. IntJ Biol Macromol 110: 167-178.

19. Gao G, Jiang YW, Jia HR, Wu FG (2019) Near-infrared light-controllable on-demand antibiotics release using thermo-sensitive hydrogel-based drug reservoir for combating bacterial infection. Biomaterials 188: 8395.
20. Wu Y, Liang J, Horkay F, Libera M (2016) Antimicrobial loading into and release from poly(ethylene glycol)/poly(acrylic acid) semiinterpenetrating hydrogels. J Polym Sci Pol Phys 54: 64-72.

21. Ono RJ,Lee Al, Chin W, Goh WS, Lee AY, et al. (2015) Biodegradable Block Copolyelectrolyte Hydrogels for Tunable Release of Therapeutics and Topical Antimicrobial Skin Treatment. ACS Macro Lett 4(9): 886-891.

22. Glisoni RJ, García Fernández MJ, Pino M, Gutkind G, Moglioni Ag (2013) Carbohyd. Polym 93: 449-457.

23. Lee Al, Venkataraman S, Fox CH, Coady DJ, Frank CW, etal. (2015) Modular composite hydrogels from cholesterol-functionalized polycarbonates for antimicrobial applications.J Mater Chem B 3: 6953-6963.

24. Shukla S, Shukla A (2018) Tunable antibiotic delivery from gellan hydrogels. J Mater Chem B 6: 6444-6458.

25. Shi Y, Truong VX, Kulkarni K, Qu Y, Simon GP, et al. (2015) A photocleavable polyprodrug-loaded wound dressing with UV-responsive antibacterial property J Mater Chem B 3: 8771-8774.

26. Wu F, Meng G, He J, Wu Y, Wu F, et al. (2014) Facile Synthesis of The Li-Rich Layered Oxide Li1.23Ni0.09Co0.12Mn0.5602 with Superior Lithium Storage Performance and New Insights into Structural Transformation of the Layered Oxide Material during Charge-Discharge Cycle: In Situ XRD Characterization. ACS Appl Mater Inter 6(8): 10005-10013.

27. Pazos E, Sleep E, Rubert Perez CM, Lee SS, Tantakitti F, et al. (2016) Nucleation and Growth of Ordered Arrays of Silver Nanoparticles on Peptide Nanofibers: Hybrid Nanostructures with Antimicrobial Properties. J. Am Chem Soci 138 (17): 5507-5510.

28. Thomas V, Yallapu MM, Sreedhar B, Bajpai SK (2007) A versatile strategy to fabricate hydrogel-silver nanocomposites and investigation of their antimicrobial activity. Colloid Interf Sci 315 (1): 389-395.

29. Yu H, Xu X, Chen X, Lu T, Zhang, et al. (2007) Preparation and antibacterial effects of PVA-PVP hydrogels containing silver nanoparticles]. Appl Polym Sci 103: 125-133.

30. Varaprasad K, Mohan YM, Ravindra S, Reddy NN, Vimala K, et al. (2010) Hydrogel-silver nanoparticle composites: A new generation of antimicrobialsJ. Appl Polym Sci 115(12): 1199-1207.

31. Xu H, Zhang G, Xu K, Wang L, Yu L, et al. (2018) Mater Sci Eng C 90: 379386.

32. Golabdar A, Adelnia H, Moshtzan N, Nasrollah Gavgani J, Izadi Vasafi H (2019) Anti-bacterial poly(vinyl alcohol) nanocomposite hydrogels reinforced with in situ synthesized silver nanoparticles. Polym Composite 40: 1322-1328.

33. Ferfera Harrar H, Berdous D, Benhalima T (2018) Polym Bull 75: 2819 2846.

34. Vimala K, Kanny K, Varaprasad K, Kumar NM, Reddy GS (2014) Novelporous-Ag0 nanocomposite hydrogels via green process for advanced antibacterial applications.J Biomed Mater Res A 102(12): 4616-4624.

35. Cui YF, Xing ZJ, Yan J, Lu YH, Xiong XQ et al. (2018) Polymers 152: 459466.

36. Sui X, Feng X, Di Luca A, Blitterswijk CA, Moroni L, (2013) Poly(Nis op ropylacrylamide)-poly(ferrocenylsilane)dual-responsive hydrogels: synthesis, characterization and antimicrobial applications. Polym Chem 4: 337-342.

37. Wang L, Li X, Sun T, Tsou YH, Chen H, et al. (2018) Dual-Functiona Dextran-PEG Hydrogel as an Antimicrobial Biomedical Material. Macromol. Biosci 18(2): 1700325.

38. Nguyen DT, Nguyen TT, Ly KL, Tran AH, Nguyen TTN, et al. (2019) Int J Polym Sci 1-10.

39. Shin JU, Gwon JY, Lee SY, Yoo HS (2018) Silver-Incorporated Nanocellulose Fibers for Antibacterial Hydrogels. ACS Omega 3: 16150-16157.

40. Hu H, Wu X, Wang H, Wang H, Zhou J (2019) Photo-reduction of Ag nanoparticles by using cellulose-based micelles as soft templates: Catalytic and antimicrobial activities. Carbohyd Polym 213: 419-427. 
41. Leawhiran N, Pavasant P, Soontornvipart K, Supaphol P (2014) Gamma irradiation synthesis and characterization of AgNP/gelatin/PVA hydrogels for antibacterial wound dressings. J Appl Polym 131: 41138.

42. Wu J, Hou S, Ren D, Mather PT (2009) Antimicrobial properties of nanostructured hydrogel webs containing silver. Biomacromolecules 10: $2686-2693$.

43. Nho YC, Lim YM, Gwon HJ, Choi EK (2010) Preparation and characterization of PVA/PVP/glycerin/antibacterial agent hydrogels using $\gamma$-irradiation followed by freeze-thawing. Korean J Chem Eng 26: 1675-1678.

44. Chang HW, Lin YS, Tsai YD, Tsai ML (2013) Effects of chitosan characteristics on the physicochemical properties, antibacterial activity, and cytotoxicity of chitosan/2-glycerophosphate/nanosilver hydrogels. J Appl Polym Sci 127: 169-176.

45. Boonkaew B, Kempf M, Kimble R, Supaphol P, Cuttle L (2014) Antimicrobial efficacy of a novel silver hydrogel dressing compared to two common silver burn wound dressings: Acticoat ${ }^{\mathrm{TM}}$ and Poly Mem Silver(®). Burns 40: 89-96.

46. Ravindra S, Mulaba-Bafubiandi AF, Rajinikanth V, Varaprasad K, Reddy NN et al. (2012) J Inorg and Organomet P 22:1254-1262.

47. Lustosa A, De Jesus Oliveira A, Quelemes P, Plácido A, Da Silva F, et. al. (2017) In Situ Synthesis of Silver Nanoparticles in a Hydrogel of Carboxymethyl Cellulose with Phthalated-Cashew Gum as a Promising Antibacterial and Healing Agent. Int J Mol Sci 18: 2399.

48. Wei QB, Fu F, Zhang YQ , Tang L(2014) J Polym Res 21: 349.

49. Uygun M, Kahveci MU, Odaci D, Timur S, Yagci Y (2009) Antibacterial Acrylamide Hydrogels Containing Silver Nanoparticles by Simultaneous Photoinduced Free Radical Polymerization and Electron Transfer Processes. Macromol Chem Phys 210: 1867-1875.

50. Lee WF, Huang YC (2007) Swelling and antibacterial properties for the superabsorbent hydrogels containing silver nanoparticles. J Appl Polym Sci 106: 1992-1999.

51. Pirtarighat S, Ghannadnia M, Baghshahi S (2019) Green synthesis of silver nanoparticles using the plant extract of Salvia spinosa grown in vitro and their antibacterial activity assessment. J Nanostructure Chem 9: $1-9$

52. Sharma B, Mahata A, Manadani S, Sarma TK, Pathak B (2016) RSC Adv 6: 2968-2973.

53. Le Thi P, Lee Y, Thi TTH, Park KM, Park KD (2018) Catechol-rich gelatin hydrogels in situ hybridizations with silver nanoparticle for enhanced antibacterial activity. Mater Sci Eng C 92: 52-60.

54. Seku K, Gangapuram BR, Pejjai B, Kadimpati KK, Golla N (2018) Microwave-assisted synthesis of silver nanoparticles and their application in catalytic, antibacterial and antioxidant activities. J Nanostructure Chem 8: 179-188.

55. Neimash VB, Kupianskyi HD, Olkhovyk IV, Styopkin VI, Lytvynchuk PM, et al. (2019) J Phys 64: 41-47.

56. Boonkaew B, Barber PM, Rengpipat S, Supaphol P, Kempf M, et al. (2014) Development and characterization of a novel, antimicrobial, sterile hydrogel dressing for burn wounds: single-step production with gamma irradiation creates silver nanoparticles and radical polymerization. J Pharm Sci 103: 3244-3253.

57. Spasojević J, Radosavljević A, Krstić J, Mitrić M, Popović M, et al. (2017) Structural characteristics and bonding environment of Ag nanoparticles synthesized by gamma irradiation within thermo-responsive poly(Nisopropylacrylamide) hydrogel. Polym Composite 38:1014-1026.

58. Yadollahi M, Namazi H,Aghazadeh M (2015) Antibacterial carboxymethyl cellulose/Ag nanocomposite hydrogels cross-linked with layered double hydroxides. Int J Biol Macromol 79: 269-277.

59. Bozaci E, Akar E, Ozdogan E, Demir A, Altinisik (2015) Application of carboxymethylcellulose hydrogel based silver nanocomposites on cotton fabrics for antibacterial property. Carbohyd Polym 134: 128-135.
60. Thomas V, Yallapu MM, Sreedhar B, Bajpai SK (2009) Breathing-in/ breathing-out approach to preparing nanosilver-loaded hydrogels: Highly efficient antibacterial nanocomposites. J Appl Polym Sci 111: 934-944.

61. Masood N, Ahmed R, Tariq M, Ahmed Z, Masoud MS, et al. (2019) Silver nanoparticle impregnated chitosan-PEG hydrogel enhances wound healing in diabetes induced rabbits. Int J Pharm559: 23-36.

62. Qin Y, Chen LL, Pu W, Liu P, Liu SX, et al. (2019) A hydrogel directly assembled from a copper metal-organic polyhedron for antimicrobial application. Chem Commun 55: 2206-2209.

63. Jones DS, Lorimer CJ, Andrews GP, Mc Coy CP, Gorman SP (2007) An examination of the thermorheological and drug release properties of zinc tetraphenylporphyrin-containing thermoresponsive hydrogels, designed as light activated antimicrobial implants.Chem Eng Sci 62: 990-999.

64. Mohamed RR, Seoudi RS, Sabaa MW (2012) Synthesis and characterization of antibacterial semi-interpenetrating carboxymethyl chitosan/poly (acrylonitrile) hydrogels. Cellulose 19: 947-958.

65. Morais DS, Rodrigues MA, Lopes MA, Coelho MJ, Maurício AC, et al. (2013) A low-cost and high-performance ball-milled Si-based negative electrode for high-energy Li-ion batteries. J Mater Sci Mater M 24: 21452155.

66. W Gao, D Vecchio, J Li, J Zhu, Q Zhang, et al. (2014) Hydrogel Containing Nanoparticle-Stabilized Liposomes for Topical Antimicrobial Delivery. ACS Nano 8(3): 2900-2907.

67. ME Villanueva, AMDR Diez, JA González, CJ Pérez, M Orrego, et al. (2016) Antimicrobial Activity of Starch Hydrogel Incorporated with Copper Nanoparticles. ACS Appl Mater Inter 8(25): 16280-16288.

68. M Yadollahi, I Gholamali, H Namazi, M Aghazadeh (2015) Synthesis and characterization of antibacterial carboxymethyl cellulose/ZnO nanocomposite hydrogels. Int J Biol Macromol 74: 136-141.

69.F Wahid, HS Wang, YS Lu, C Zhong, LQ Chu (2017) Preparation, characterization and antibacterial applications of carboxymethyl chitosan/CuO nanocomposite hydrogels. Int J Biol Macromol 101: 690695.

70. Rakhshaei R, Namazi H (2017) A potential bioactive wound dressing based on carboxymethyl cellulose/ZnO impregnated MCM-41 nanocomposite hydrogel. Mat Sci Eng C 73: 456-464.

71. Straccia MC, d'Ayala GG, Romano I, Laurienzo P (2015) Novel zinc alginate hydrogels prepared by internal setting method with intrinsic antibacterial activity. Carbohydr Polym 125: 103-112.

72. W Gao, CM Hu, RH Fang, L Zhang (2013Liposome-like nanostructures for drug delivery. J Mater Chem B 1: 6569-6585.

73. GF Mehyar, Z Liu, JH Han (2008) Dynamics of antimicrobial hydrogels in physiological saline. Carbohyd Polym 74: 92-98.

74. EA Bursali, S Coskun, M Kizil, M Yurdakoc (2011) Synthesis, characterization and in vitro antimicrobial activities of boron/starch/ polyvinyl alcohol hydrogels. Carbohyd Polym 83(3): 1377-1383.

75. SJ Fallows, MJ Garland, CM Cassidy, MM Tunney, TRR Singh, et al. (2012) Electrically-responsive anti-adherent hydrogels for photodynamic antimicrobial chemotherapy. J Photoch Photobiol B 114: 61-72.

76. SP Tan, P Mc Loughlin, L O’Sullivan, ML Prieto, GE Gardiner, et al. (2013) Development of a novel antimicrobial seaweed extract-based hydrogel wound dressing. Int J Pharm 456(1): 10-20.

77. X Wang, Z Liu, X Ye, K Hu, H Zhong, et al. (2015) A facile one-pot method to two kinds of graphene oxide-based hydrogels with broad-spectrum antimicrobial properties. Chem Eng J 260: 331-337.

78. H Kitagawa, K Takeda, R Kitagawa, N Izutani, S Miki, et al. (2014) Development of sustained antimicrobial-release systems using poly(2hydroxyethyl methacrylate)/trimethylolpropane trimethacrylate hydrogels. Acta biomater 10(10): 4285-4295. 
79. D Jeong, HK Kim, JP Jeong, SD Dindulkar, E Cho, et al. (2016) Cyclosophoraose/cellulose hydrogels as an efficient delivery system for galangin, a hydrophobic antibacterial drug. Cellulose 23: 2609-2625.

80. RT Cleophas, J Sjollema, HJ Busscher, JA Kruijtzer, RM Liskamp (2014) Characterization and Activity of an Immobilized Antimicrobial Peptide Containing Bactericidal PEG-Hydrogel. Biomacromolecules 15(9): 3390-3395.

81. J Li, R Xing, S Bai, X Yan (2019) Recent advances of self-assembling peptide-based hydrogels for biomedical applications. Soft Matter 15: 1704-1715.

82. N Annabi, D Rana, ES Sani, R Portillo Lara, JL Gifford, et al. (2017) Engineering a sprayable and elastic hydrogel adhesive with antimicrobial properties for wound healing. Biomaterials 139: 229-243.

83. Z Xie, NV Aphale, TD Kadapure, AS Wadajkar, S Orr, et al. (2015) Design of antimicrobial peptides conjugated biodegradable citric acid derived hydrogels for wound healing. J Biomed Mater Res A 103(12): 3907-3918.

84. G Yang, T Huang, Y Wang, H Wang, Y Li, et al. (2018) Sustained Release of Antimicrobial Peptide from Self-Assembling Hydrogel Enhanced Osteogenesis. J Biomat Sci Polym E 29(15): 1812-1824.

85. A Ali, S Ahmed (2018) A review on chitosan and its nanocomposites in drug delivery. Int J Biol Macromol 109: 273-286.

86. RA Muzzarelli (1996) Carbohyd Polym 29: 309-316.

87. E Guibal (2004) Interactions of metal ions with chitosan-based sorbents: a review. Sep Purif Technol 38(1): 43-74

88. JM Wu, X Cheng, YL Li, GS Yang (2019) Carbohyd Polym 211: 152-160.

89. AS Montaser, FA Mahmoud (2019) Preparation of Chitosan-graftedPolyvinyl acetate Metal Nanocomposite for producing Multifunctional Textile Cotton Fabrics. Int J Biol Macromol 124: 659-666.

90. M Kong, XG Chen, K Xing, HJ Park (2010) Antimicrobial properties of chitosan and mode of action: A state of the art review. Int J Food Microbiol 144(1): 51-63.

91. NA Mohamed, MM Fahmy (2012) Synthesis and Antimicrobial Activity of Some Novel Cross-Linked Chitosan Hydrogels. Int J Mol Sci 13(9): 11194-11209.

92. NA Mohamed, NF Al Harby, MS Almarshed (2018) Synthesis and characterization of novel trimellitic anhydride isothiocyanate-cross linked chitosan hydrogels modified with multi-walled carbon nanotubes for enhancement of antimicrobial activity. Int J Biol Macromol 132: 416428.

93. NA Mohamed, NAA El Ghany, MM Fahmy (2016) Novel antimicrobia superporous cross-linked chitosan/pyromellitimide benzoyl thiourea hydrogels. Int J Biol Macromol 82: 589-598.

94. F Ding, Z Nie, H Deng, L Xiao, Y Du, X Shi (2013) Antibacterial hydroge coating by electrophoretic co-deposition of chitosan/alkynyl chitosan. Carbohyd Polym 98(2): 1547-1552.

95. Zhao X, Wu H, Guo B, Dong R, Qiu Y, et al. (2017) Antibacterial antioxidant electroactive injectable hydrogel as self-healing wound dressing with hemostasis and adhesiveness for cutaneous wound healing. Biomaterials 122: 34-47.

96. W Zou, Y Chen, X Zhang, J Li, L Sun, et al. (2018) Carbohyd Polym 202 246-257.

97. P Li, YF Poon, W Li, HY Zhu, SH Yeap, et al. (2011) A polycationic antimicrobial and biocompatible hydrogel with microbe membrane suctioning ability. Nat Mater 10: 149-156.

98. N Peng, Y Wang, Q Ye, L Liang, Y An, et al. (2016) Carbohyd Polym 137: 59-64.

99. H Wang, G Zha, H Du, L Gao, X Li, et al. (2014) Facile fabrication of ultrathin antibacterial hydrogel films via layer-by-layer "click" chemistry. Polym Chem 5: 6489-6494.
100. H Du, G Zha, L Gao, H Wang, X Li, et al. (2014) Fully biodegradable antibacterial hydrogels via thiol-ene "click" chemistry. Polym Chem 5 4002-4008.

101. C Zhou, VX Truong, Y Qu, T Lithgow, G Fu, et al. (2016) Antibacterial poly(ethylene glycol) hydrogels from combined epoxy-amine and thiol-ene click reaction. Polym Sci Poly Chem 54(5): 656-667.

102. OCJ Andrén, T Ingverud, D Hult, J Håkansson, Y Bogestål, et al. (2019) Antibiotic-Free Cationic Dendritic Hydrogels as Surgical-Site-InfectionInhibiting Coatings. Adv Healthcare Mater 8(5): 1801619.

103. A Zheng, Y Xue, D Wei, S Li, H Xiao, et al. (2014) Synthesis and Characterization of Antimicrobial Polyvinyl Pyrrolidone Hydrogel as Wound Dressing. Soft Mater 12(3): 179-187.

104. BD Krezović, SI Dimitrijević, JM Filipović, RR Nikolić, SL Tomić (2012) Antimicrobial P(HEMA/IA)/PVP semi-interpenetrating network hydrogels. Polym Bull 70: 809-819.

105. R Wang, DL Xu, L Liang, TT Xu, W Liu, et al. (2016) Enzymatically crosslinked epsilon-poly-l-lysine hydrogels with inherent antibacterial properties for wound infection prevention. RSC Adv 6: 8620-8627.

106. Sahiner N, Sagbas S, Sahiner M, Silan C, Aktas N (2016) Biocompatible and biodegradable poly(Tannic Acid) hydrogel with antimicrobial and antioxidant properties. Int J Biol Macromol 82: 150-159.

107. AL Lee, VW Ng, W Wang, JL Hedrick, YY Yang (2013) Block copolymer mixtures as antimicrobial hydrogels for biofilm eradication. Biomaterials 34(38): 10278-10286.

108. A Pascual, JP Tan, A Yuen, JM Chan, DJ Coady, et al. (2015) BroadSpectrum Antimicrobial Polycarbonate Hydrogels with Fast Degradability. Biomacromolecules 16(4): 1169-1178.

109. Q Liu, L. Liu (2018) Novel Light-Responsive Hydrogels with Antimicrobial and Antifouling Capabilities. Langmuir 35(5): 14501457.

110. DA Salick, JK Kretsinger, DJ Pochan, JP Schneider (2007) Inherent antibacterial activity of a peptide-based beta-hairpin hydrogel. J Am Chem Soci 129(47): 14793-14799.

111. DA Salick, DJ Pochan, JP Schneider (2009) Design of an Injectable $\beta$-Hairpin Peptide Hydrogel That Kills Methicillin-Resistant Staphylococcus aureus. Adv Mater 21(41): 4120-4123.

112. S Roy, PK Das (2008) Antibacterial hydrogels of amino acid-based cationic amphiphiles. Biotechnol Bioeng 100(4): 756-764.

113. K Malhotra, S Shankar, R Rai, Y Singh (2018) Broad-Spectrum Antibacterial Activity of Proteolytically Stable Self-Assembled $\alpha \gamma$ Hybrid Peptide Gels. Biomacromolecules19(3): 782-792.

114. Marchesan S, Qu Y, Waddington LJ, Easton CD, Glattauer V, et al. (2013) Self-assembly of ciprofloxacin and a tripeptide into an antimicrobial nanostructured hydrogel. Biomaterials 34: 3678-3687.

115. Irwansyah I, Li YQ Shi W, Qi D, Leow WR, et al. (2015) Gram-Positive Antimicrobial Activity of Amino Acid-Based Hydrogels. Adv Mater 27: 648-654.

116. Tahtat D, Mahlous M, Benamer S, Khodja AN, Youcef SL, et al. (2011) Influence of some factors affecting antibacterial activity of PVA/ Chitosan based hydrogels synthesized by gamma irradiation. J Mater Sci Mater M 22: 2505-2512.

117. Cao A, Tang Y, Liu Y, Yuan H, Liu L (2013) A surfactant-assisted redox hydrothermal route to prepare highly photoluminescent carbon quantum dots with aggregation-induced emission enhancement properties. Chem Commun 49: 5574-5576.

118. Sudarsan S, Franklin DS, Sakthivel M, Guhanathan S (2016) Nontoxic antibacterial, biodegradable hydrogels with $\mathrm{pH}$-stimuli sensitivity: investigation of swelling parameters. Carbohyd Polym 148: 206-215.

119. Fang H, Wang J, Li l, Xu L, Wu Y, et al. (2019) A novel high-strength poly (ionic liquid)/PVA hydrogel dressing for antibacterial applications. Chem Eng J 365: 153-164. 
120. Giano MC, Ibrahim Z, Medina SH, Sarhane KA, Christensen JM, et al. (2014) Injectable bioadhesive hydrogels with innate antibacterial properties. Nat Commun 5: 4095.

121. Bedel NS, Tezcan M, Ceylan O, Gurdag G, Cicek H (2015) Effects of pore morphology and size on antimicrobial activity of chitosan/ poly(ethylene glycol) diacrylate macromer semi-IPN hydrogels. J Appl Polym Sci 132: 42707.

122. O'Connor NA, Abugharbieh A, Yasmeen F, Buabeng E, Mathew S, et al. (2015) The crosslinking of polysaccharides with polyamines and dextran-polyallylamine antibacterial hydrogels. Int J Biol Macromol 72: 88-93.

123. Kaith BS, Sharma R, Kalia S (2015) Polymer Nanocomposites in Biomedical Engineering. Int J Biol Macromol 75: 266-275.

124. Fan L, Yang J, Wu H, Hu Z, Yi J, et al. (2015) Preparation and characterization of quaternary ammonium chitosan hydrogel with significant antibacterial activity. Int J Biol Macromol 79: 830-836.

\section{ISSN: 2574-1241}

DOI: 10.26717/BJSTR.2019.23.003973

Na Peng. Biomed J Sci \& Tech Res

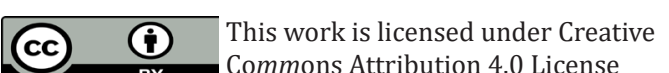

Submission Link: https://biomedres.us/submit-manuscript.php
125. Singh D, Singh A, Singh R (2015) Polyvinyl pyrrolidone/carrageenan blend hydrogels with nano silver prepared by gamma radiation for use as an antimicrobial wound dressing. J Biomat Sci Poly E 26: 1269-1285.

126. Xiao Y, Gong T, Jiang Y, Wang Y, Wen ZT, et al. (2016) Fabrication and characterization of a glucose-sensitive antibacterial chitosanpolyethylene oxide hydrogel. Polymer 82: 1-10.

127. Khamrai M, Banerjee SL, Paul S, Samanta S, Kundu PP (2019) Curcumin entrapped gelatin/ionically modified bacterial cellulose based selfhealable hydrogel film: An eco-friendly sustainable synthesis method of wound healing patch. Int J Biol Macromol 122: 940-953.

128. Imtiaz N, Niazi MBK, Fasim F, Khan sBA, Bano SA, et al. (2019) Int J Polym Sci p. 1-11.

129. Chuah C, Wang J, Tavakoli J, Tang Y (2018) Novel Bacterial CellulosePoly (Acrylic Acid) Hybrid Hydrogels with Controllable Antimicrobial Ability as Dressings for Chronic Wounds. Polymers 10(12): 1323.

$\begin{array}{ll}\text { BIOMEDICAL } & \text { Assets of Publishing with us } \\ \text { RESEARCHES } & \text { - Global archiving of articles } \\ & \text { - Immediate, unrestricted online access } \\ & \text { - Rigorous Peer Review Process } \\ & \text { - Authors Retain Copyrights } \\ \end{array}$

